\title{
Estimation of Total Costs in Pediatric and Young Adult Patients with Relapsed or Refractory Acute Lymphoblastic Leukemia Receiving Tisagenlecleucel from a U.S. Hospital's Perspective
}

\author{
Hongbo Yang, PhD; Yanni Hao, PhD; Cynthia Z. Qi, MBA; Xinglei Chai, PhD; and Eric Q. Wu, PhD
}

\begin{abstract}
BACKGROUND: Tisagenlecleucel was approved for the treatment of pediatric and young adult patients with relapsed/refractory $(r / r) B$-cell acute lymphoblastic leukemia (ALL) based on the pivotal ELIANA trial.

OBJECTIVE: To comprehensively evaluate the total costs associated with tisagenlecleucel treatment, including costs from pre- to postinfusion periods of tisagenlecleucel in addition to the cost of tisagenlecleucel.

METHODS: An economic model was developed to estimate total costs associated with tisagenlecleucel treatment from the time of leukapheresis to 2 months postinfusion from a U.S. hospital's perspective. Costs were estimated based on resource use and safety management from the ELIANA trial and were considered during the pretreatment, tisagenlecleucel infusion, and follow-up periods of treatment. Cost components included leukapheresis, lymphodepleting chemotherapy, tisagenlecleucel infusion and hospital administration, inpatient and intensive care unit admissions, medical professional visits, laboratory tests and procedures, and management of major adverse events. Scenario analyses were conducted by varying key assumptions related to adverse events and hospitalization.

RESULTS: The total cost associated with tisagenlecleucel treatment among pediatric and young adult patients with r/r ALL was estimated to be $\$ 612,779$, of which $\$ 137,636(22.5 \%)$ was in addition to the list price of tisagenlecleucel $(\$ 475,000)$ and the associated administration cost of $\$ 143.08$. The top 3 drivers of the additional cost were adverse event management $(\$ 70,968 ; 51.6 \%)$, inpatient and intensive care unit admissions not attributed to adverse events $(\$ 57,952 ; 42.1 \%)$, and laboratory tests and procedures $(\$ 5,209 ; 3.8 \%)$. The costs incurred during the pretreatment, infusion, and follow-up periods were $\$ 29,002, \$ 476,659$, and $\$ 107,118$, respectively. In the scenario analyses, the total costs ranged from $\$ 483,169$ (tisagenlecleucel treatment in the outpatient setting without adverse events) to $\$ 672,373$ (tisagenlecleucel treatment in the inpatient setting with grade $3 / 4$ cytokine release syndrome and B-cell aplasia).
\end{abstract}

CONCLUSIONS: In this economic model, tisagenlecleucel treatment among pediatric and young adult patients with $\mathrm{r} / \mathrm{r}$ ALL was estimated to cost $\$ 612,779$. The cost of care in addition to the price of tisagenlecleucel accounted for $22.5 \%$ of the total, with adverse event management and inpatient and intensive care unit admissions being main drivers. Further studies are warranted to assess the cost of tisagenlecleucel treatment in the context of current standards of care in real-world clinical practice.

J Manag Care Spec Pharm. 2020;26(8):971-80

Copyright @ 2020, Academy of Managed Care Pharmacy. All rights reserved.

\section{What is already known about this subject}

Tisagenlecleucel is a CD19-directed chimeric antigen receptor T-cell therapy that is approved for the treatment of pediatric and young adult patients with relapsed/refractory B-cell acute lymphoblastic leukemia (ALL).

Tisagenlecleucel is priced at $\$ 475,000$, which does not include the cost of associated resource use and adverse event management.

There is limited real-world evidence to inform patterns of health care resource utilization related to tisagenlecleucel treatment and adverse event management; however, comprehensive resource utilization data were collected in the pivotal ELIANA trial.

\section{What this study adds}

In this economic model, we estimated a total cost of $\$ 612,779$ associated with tisagenlecleucel treatment from leukapheresis to 2 months postinfusion among pediatric and young adult patients with relapsed/refractory ALL, of which $\$ 137,636$ (22.5\%) was in addition to the list price of tisagenlecleucel $(\$ 475,000)$ and the associated administration cost (\$143.08).

The top 3 drivers of the additional cost were adverse event management (51.6\%), inpatient and intensive care unit admissions not attributed to adverse events (42.1\%), and laboratory tests and procedures (3.8\%).

A cute lymphoblastic leukemia (ALL) is a hematological malignancy that primarily affects children, adolescents, and young adults, comprising more than 25\% of pediatric cancer diagnoses in the United States. ${ }^{1,2}$ While the majority of patients with ALL achieve remission after frontline therapy, relapse and refractoriness remain a challenge and are associated with poor clinical outcomes. ${ }^{3}$

Historically, patients with refractory disease or those who experience 2 or more relapses after treatment with chemotherapy have especially poor prognoses and dismal survival outcomes, with median overall survival (OS) ranging between 3 and 7.5 months. ${ }^{4-6}$ Conventional treatment options include salvage chemotherapy, such as clofarabine monotherapy or combination therapy with cyclophosphamide or etoposide, and blinatumomab, followed by allogeneic hematopoietic stem cell transplantation (HSCT). ${ }^{7}$ However, these treatment options 
have limited efficacy, with median OS of fewer than 10 months and objective response rates of less than $50 \% .5,6,8,9$

Recent therapeutic advances within the ALL treatment landscape have led to the introduction of chimeric antigen receptor T-cell (CAR-T) immunotherapy, which involves the genetic modification of a patient's own T cells to target cancer cells. Tisagenlecleucel, a CD19-directed CAR-T therapy, was recently approved by the U.S. Food and Drug Administration for the treatment of pediatric and young adult patients with relapsed/refractory ( $\mathrm{r} / \mathrm{r}$ ) B-cell ALL, based on its efficacy and safety evaluated in the ELIANA clinical trial. ${ }^{10,11}$ In this global, phase 2 , single-arm study, pediatric and young adult patients with $\mathrm{r} / \mathrm{r}$ ALL treated with a single infusion of tisagenlecleucel achieved promising outcomes compared with current standard therapies, with a median OS of 19.1 months and an overall remission rate of $81 \%$ within 3 months. ${ }^{10}$

As a form of personalized medicine with demonstrated clinical outcomes, tisagenlecleucel has been priced at $\$ 475,000$ based on wholesale acquisition in the United States. However, because tisagenlecleucel is the first-in-class CAR-T immunotherapy and is individually manufactured for each patient using their own blood cells, the associated resource use could be very different from other cancer treatments. Indeed, before the manufacturing and infusion of tisagenlecleucel, apheresis is required to cultivate the patient's cells for the manufacturing process. ${ }^{11}$ Subsequently, bridging chemotherapy treatments and lymphodepleting regimens may also be needed to stabilize the patient's condition in preparation for tisagenlecleucel infusion. ${ }^{10,12}$ Moreover, since tisagenlecleucel is designed to use a patient's own immune system to eliminate cancer cells, treatment is also associated with unique adverse events (AEs), including cytokine release syndrome (CRS) and B-cell aplasia. ${ }^{11}$ Both of these AEs may need management with expensive therapies (i.e., tocilizumab and intravenous immunoglobulin [IVIG]), and patients who develop severe CRS may also need to be admitted to the intensive care unit (ICU).

Importantly, there is limited real-world evidence to inform patterns of health care resource utilization (HCRU) related to treatment and AE management, since CAR- T therapy is relatively new to the market. However, comprehensive HCRU data were collected in the pivotal ELIANA trial and included information related to hospitalization and ICU stays, as well as treatment utilization for bridging chemotherapy, lymphodepletion, CRS, and B-cell aplasia.

Therefore, this study was conducted to comprehensively evaluate the total costs of tisagenlecleucel treatment, including costs additional to the procedure and from multiple treatment periods, using the latest clinical trial data from the ELIANA study and publicly available data. We also performed scenario analyses to assess the effect on total costs based on varying hospital treatment settings, presence of AEs, cost assumptions, and data input sources. These results aim to provide valuable insight into the cost of care associated with tisagenlecleucel therapy and to fill the current knowledge gaps within the economic literature of CAR-T therapy.

\section{Methods}

\section{Model Overview}

An economic model was developed in Excel (Microsoft, Redmond, WA) to assess the total costs associated with tisagenlecleucel treatment among pediatric and young adult patients with $\mathrm{r} / \mathrm{r}$ ALL from a U.S. hospital's perspective. The total costs were estimated from the time of leukapheresis to 2 months postinfusion, which was consistent with the time frame of collecting HCRU related to the tisagenlecleucel infusion per the trial protocol. The model was developed using a fee-for-service approach, which estimated costs based on the HCRU and safety data from the pivotal, phase 2 ELIANA trial. ${ }^{10,13}$

The model considered costs incurred during 3 treatment periods: (a) the pretreatment period, during which $\mathrm{T}$ cells were harvested from patients and preparative lymphodepleting chemotherapy was given; (b) the infusion period (i.e., the day of infusion); and (c) the follow-up period, during which patients were monitored or treated for disease progression or AEs for up to 2 months (Figure 1). The cost components included leukapheresis, lymphodepleting chemotherapy, tisagenlecleucel infusion and hospital administration, inpatient and ICU admission, medical professional visits, laboratory tests and procedures, and additional medication and HCRU for the management of major AEs (e.g., grade 3/4 CRS and B-cell aplasia). All costs were inflation-adjusted to 2019 U.S. dollars (USD).

The base-case model estimated the total costs using the observed hospitalization, ICU, and AE data for all patients receiving tisagenlecleucel infusion in the ELIANA trial. ${ }^{10,13}$ Scenario analyses were conducted by varying key inputs and assumptions. Specifically, scenario analyses included: (a) assuming all patients were treated with tisagenlecleucel in the outpatient setting and did not experience any AEs during follow-up; (b) assuming all patients were treated with tisagenlecleucel in the inpatient setting and experienced grade 3/4 CRS and B-cell aplasia during follow-up; (c) considering the variation of individual cost components (i.e., lymphodepleting regimen and administration costs, inpatient and ICU admission costs, medical professional visit costs, laboratory test and procedure costs, and $\mathrm{AE}$ costs) within a 95\% confidence interval (CI) or $\pm 25 \%$ if $95 \%$ CI was not estimable; (d) using Grupp et al. (2019), a study based on real-world use of tisagenlecleucel, to inform grade 3/4 CRS, IVIG use, and tocilizumab use (Appendix A, available in online article) ${ }^{14}$. and (e) estimating the daily hospitalization and ICU costs based on an alternative data source-the Healthcare Cost and Utilization Project (HCUP) database..$^{15,16}$ 


\section{FIGURE 1 Overview of the HCRU Cost Model for Patients with r/r ALL Receiving Tisagenlecleucel}

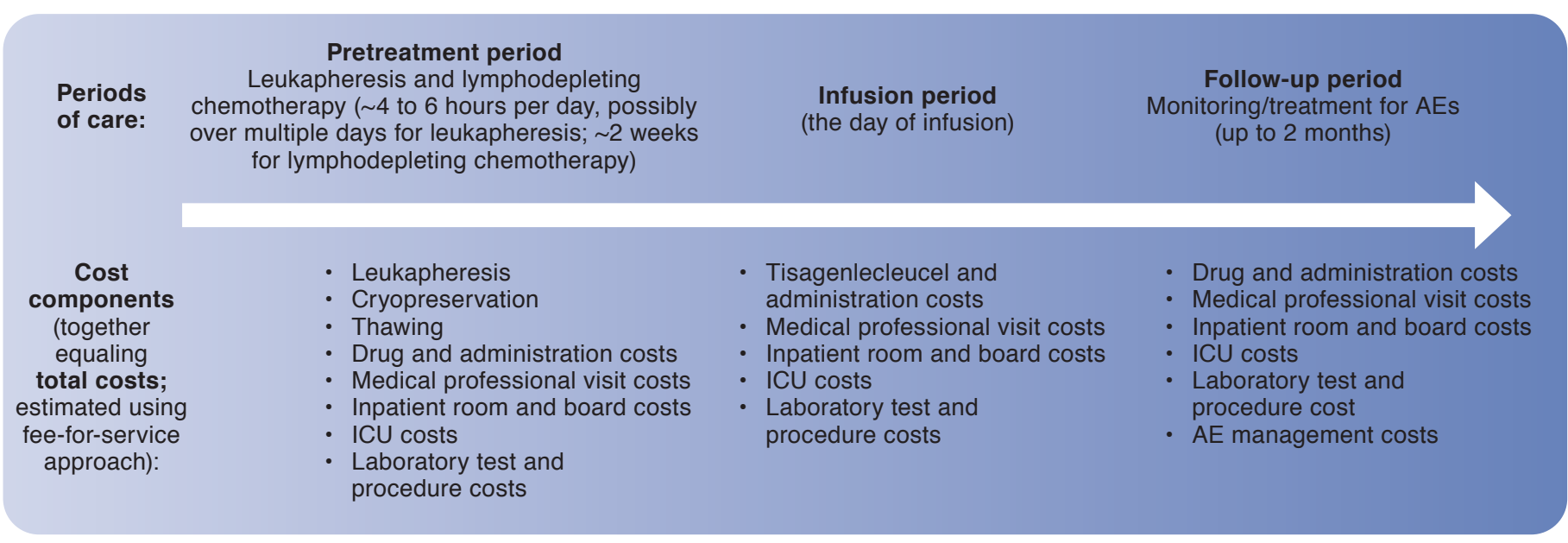

$A E=$ adverse event; $A L L=$ acute lymphoblastic leukemia; $H C R U=$ health care resource utilization; $I C U=$ intensive care unit; $r / r=r e l a p s e d /$ refractory.

\section{Model Assumptions}

This model considered the period of care starting from cell collection to 2 months post-tisagenlecleucel infusion.

This model used the observed hospitalization data based on the ELIANA trial, which indicated that some patients received tisagenlecleucel in the inpatient setting and some in the hospital outpatient setting. ${ }^{10,13}$ The model separately estimated costs associated with AEs (including drug, procedure, and hospitalization costs) and costs associated with inpatient and ICU admissions because of causes other than AEs.

\section{Model Inputs}

Lymphodepleting Regimens and Administration Costs. Costs associated with lymphodepleting chemotherapies were considered during the pretreatment period. As estimated from the ELIANA trial data, $96 \%$ of the infused population received lymphodepleting therapies (95\% received regimen 1 [fludarabine and cyclophosphamide] and 1\% received regimen 2 [cytarabine and etoposide]) during the pretreatment period. ${ }^{13}$ The remaining $4 \%$ of patients did not receive lymphodepleting therapies, as observed in the ELIANA trial.

The default cost inputs for medication and administration were obtained from the public domain and were based on payment from the payers (i.e., costs reimbursed by payers). Specifically, medication costs were estimated using the wholesale acquisition cost (WAC) from RED BOOK. ${ }^{17}$ Unit costs for drug administration were obtained from the Centers for Medicare $\&$ Medicaid Services (CMS) Physician Fee Schedule. ${ }^{18}$ Detailed information regarding the drug costs can be found in Table 1 .
Tisagenlecleucel Drug and Administration Costs. Tisagenlecleucel drug and administration costs were considered during the infusion period. Similar to the lymphodepleting costs, the cost of the tisagenlecleucel infusion procedure was obtained based on the WAC in the RED BOOK, and the administration cost was derived from the CMS Physician Fee Schedule..$^{17,18}$

Inpatient and ICU Costs Not Attributed to AEs. Inpatient and ICU costs were estimated during the entire treatment period. The inpatient and ICU admission proportions and length of stay were estimated from the ELIANA trial data, separately for the pretreatment, infusion, and follow-up periods. ${ }^{10,13}$ Only hospitalization not attributed to AEs was considered to avoid double counting with the AE management costs. The unit hospitalization costs were obtained from the literature. The average daily hospitalization cost was obtained from Kaul et al. (2016), and the average daily ICU cost was obtained from Hsu et al. (2015). ${ }^{19,20}$ The model inputs are presented in Table 2.

Medical Professional Visit Costs. Costs associated with medical professional visits were relevant to the entire treatment period and were thus considered in the model. Resource utilization frequencies were acquired from the ELIANA trial protocol, while the unit costs of medical professional visits were obtained from the CMS Physician Fee Schedule. 10,13,18 Table 2 summarizes the detailed costs associated with medical professional visits.

Laboratory and Procedure Costs. The cost of leukapheresis was considered during the pretreatment period, and other laboratory and procedure costs were considered throughout the tisagenlecleucel treatment period. The frequency inputs of 


\begin{tabular}{|c|c|c|}
\hline Cost Item & Inputs & Source/Notes \\
\hline \multicolumn{3}{|c|}{ Pretreatment period: lymphodepleting therapy } \\
\hline Regimen 1 & $\begin{array}{l}\text { Proportion of patients }{ }^{a} \text { : } \\
94.94 \%\end{array}$ & ELIANA trial ${ }^{10,13}$ \\
\hline \multirow[t]{2}{*}{ Fludarabine } & Drug cost: $\$ 281.23$ & RED BOOK ${ }^{17}$ \\
\hline & $\begin{array}{l}\text { Administration cost: } \\
\$ 572.32\end{array}$ & $\begin{array}{l}\text { CMS Physician Fee } \\
\text { Schedule }^{18}\end{array}$ \\
\hline \multirow[t]{2}{*}{ Cyclophosphamide } & Drug cost: $\$ 817.47$ & RED BOOK ${ }^{17}$ \\
\hline & $\begin{array}{l}\text { Administration cost: } \\
\$ 138.40\end{array}$ & $\begin{array}{l}\text { CMS Physician Fee } \\
\text { Schedule }{ }^{18}\end{array}$ \\
\hline Regimen 2 & $\begin{array}{l}\text { Proportion of patients }{ }^{\mathrm{a}} \text { : } \\
1.27 \%\end{array}$ & ELIANA trial ${ }^{10,13}$ \\
\hline \multirow[t]{2}{*}{ Cytarabine } & Drug cost: $\$ 11.40$ & RED BOOK ${ }^{17}$ \\
\hline & $\begin{array}{l}\text { Administration cost: } \\
\$ 138.40\end{array}$ & $\begin{array}{l}\text { CMS Physician Fee } \\
\text { Schedule }^{18}\end{array}$ \\
\hline \multirow[t]{2}{*}{ Etoposide } & Drug cost: $\$ 42.40$ & RED BOOK ${ }^{17}$ \\
\hline & $\begin{array}{l}\text { Administration cost: } \\
\$ 522.21\end{array}$ & $\begin{array}{l}\text { CMS Physician Fee } \\
\text { Schedule }^{18}\end{array}$ \\
\hline \multicolumn{3}{|c|}{ Infusion period: tisagenlecleucel infusion } \\
\hline \multirow[t]{2}{*}{ Tisagenlecleucel } & Drug cost: $\$ 475,000$ & RED BOOK ${ }^{17}$ \\
\hline & $\begin{array}{l}\text { Administration cost: } \\
\$ 143.08\end{array}$ & $\begin{array}{l}\text { CMS Physician Fee } \\
\text { Schedule }^{18}\end{array}$ \\
\hline \multicolumn{3}{|c|}{$\begin{array}{l}\text { aThe proportion of patients was estimated based on the observation from the } \\
\text { ELIANA trial. } \\
\text { CMS = Centers for Medicare } \& \text { Medicaid Services. }\end{array}$} \\
\hline
\end{tabular}

laboratory tests and procedures during each treatment period were based on the ELIANA trial protocol. ${ }^{10,13}$ The physician cost associated with leukapheresis and the unit costs of laboratory tests and procedures were derived from the CMS Physician Fee Schedule and Clinical Laboratory Fee Schedule, respectively. ${ }^{18,21}$ The laboratory tests and procedures associated with ALL included bone marrow biopsy and/or aspirate, chemistry panel, coagulation panel, echocardiogram, electrocardiogram, hematology panel, influenza A and B test, peripheral blood collection for flow cytometry, pulse oximetry, serum cytokines, serum immunoglobulin levels, urinalysis, and other laboratory tests of special interest.

AE Costs. AE rates during the follow-up period were obtained from the ELIANA trial data, and only grade 3 and 4 AEs with rates $\geq 5 \%$ were considered in the model. ${ }^{10,13}$ Resource use was modeled separately for the 2 most important tisagenlecleucelrelated AEs, grade 3/4 CRS and B-cell aplasia, with observed rates of $48 \%$ and $61 \%$, respectively. For patients with grade 3/4 CRS, the proportions of patients with an ICU admission and tocilizumab treatment, as well as the dosage used for CRS management, were estimated based on ELIANA trial data. ${ }^{10,13}$ In the ELIANA trial, CRS was graded based on the Penn criteria, which tend to assign a higher grade of CRS compared with the latest criteria recommended by the American Society for Transplantation and Cellular Therapy (ASTCT). ${ }^{22}$ Similarly, for patients with B-cell aplasia, the proportion of patients with IVIG use, the dosing schedule, and the number of doses was also estimated based on ELIANA trial data. ${ }^{10,13}$ Unit costs for other grade $3 / 4$ AEs were derived from the HCUP Kids' Inpatient Database. ${ }^{23}$ Of note, grade 3/4 AEs included in the model were AEs regardless of study drug relationship, not restricted to those that were suspected to be study drug-related. The AE inputs are summarized in Table 3 and Appendix B (available in online article).

\section{Results}

\section{Base-Case Results}

The overall cost associated with tisagenlecleucel treatment from leukapheresis to 2 months post infusion in patients with $\mathrm{r} / \mathrm{r}$ ALL was estimated to be $\$ 612,779$ (Table 4). Considering a list price of tisagenlecleucel of $\$ 475,000$ and the associated administration cost of $\$ 143.08$, the model calculated an additional cost of care of $\$ 137,636$, which included $\$ 70,968$ (51.6\%) for AE management, $\$ 57,952$ (42.1\%) for inpatient and ICU admissions not attributed to AEs, \$5,209 (3.8\%) for laboratory tests and procedures, $\$ 1,780$ (1.3\%) for medical professional visits, and $\$ 1,727$ (1.3\%) for lymphodepleting drugs and their administration.

The costs incurred during the pretreatment period, infusion period, and follow-up period were $\$ 29,002, \$ 476,659$, and $\$ 107,118$, respectively. The primary cost drivers were $\$ 23,966$ for inpatient and ICU admissions (82.6\%) during the pretreatment period, $\$ 475,143$ for the tisagenlecleucel procedure and administration (99.7\%) during the infusion period, and $\$ 70,968$ for AE management (66.3\%) during the follow-up period.

\section{Scenario Analysis Results}

In the scenario analyses, the total costs ranged from $\$ 483,169$ (outpatient setting without AEs) to $\$ 672,373$ (inpatient setting with grade 3/4 CRS and B-cell aplasia; Table 4). Under the scenario that all patients received tisagenlecleucel treatment in the outpatient setting and did not experience any AEs during follow-up, the model calculated a cost of $\$ 8,026$ aside from the tisagenlecleucel procedure and administration, which included $\$ 5,209$ (64.9\%) for laboratory tests and procedures, $\$ 1,727$ (21.5\%) for lymphodepleting drugs and their administration, and $\$ 1,090$ (13.6\%) for medical professional visits.

Under the scenario that all patients received tisagenlecleucel treatment in the inpatient setting and experienced grade 3/4 CRS and B-cell aplasia, the model calculated a cost of $\$ 197,230$ aside from the tisagenlecleucel procedure and administration, which included $\$ 104,722$ (53.1\%) for AE management, $\$ 83,395$ (42.3\%) for inpatient and ICU admissions not attributed to AEs, \$5,209 (2.6\%) for laboratory tests and procedures, $\$ 2,177$ (1.1\%) for medical professional visits, and $\$ 1,727$ (0.9\%) for lymphodepleting drugs and their administration. 
Estimation of Total Costs in Pediatric and Young Adult Patients with Relapsed or

Refractory Acute Lymphoblastic Leukemia Receiving Tisagenlecleucel from a U.S. Hospital's Perspective

\section{TABLE 2 HCRU Cost Inputs}

\begin{tabular}{|c|c|c|}
\hline Cost Item & Inputs & Source/Notes \\
\hline \multicolumn{3}{|l|}{ Pretreatment period } \\
\hline Inpatient room and board & $\begin{array}{l}\text { Proportion of patients hospitalized: } 67.09 \% \\
\text { Average LOS: } 20.2 \text { days } \\
\text { Daily hospitalization cost: } \$ 1,323.30\end{array}$ & $\begin{array}{l}\text { ELIANA triallo,13 } \\
\text { Kaul et al. }(2016)^{19}\end{array}$ \\
\hline ICU & $\begin{array}{l}\text { Proportion of patients admitted: } 3.80 \% \\
\text { Average LOS: } 30.7 \text { days } \\
\text { Daily ICU cost: } \$ 5,217.32\end{array}$ & $\begin{array}{l}\text { ELIANA trial }{ }^{10,13} \\
\text { Hsu et al. }(2015)^{20}\end{array}$ \\
\hline Physician visit & $\begin{array}{l}\text { Average number of visits: } 2 \\
\text { Unit cost: } \$ 51.90\end{array}$ & $\begin{array}{l}\text { ELIANA trial }{ }^{10,13} \\
\text { CMS Physician Fee Schedule }{ }^{18}\end{array}$ \\
\hline Nurse visit & $\begin{array}{l}\text { Average number of visits: } 14 \\
\text { Unit cost: } \$ 25.95\end{array}$ & $\begin{array}{l}\text { ELIANA trial }{ }^{10,13} \\
\text { CMS Physician Fee Schedule }{ }^{18}\end{array}$ \\
\hline Leukapheresis & Cost: $\$ 2,012.80$ & CMS Physician Fee Schedule $^{18}$ \\
\hline $\begin{array}{l}\text { Other laboratory tests and } \\
\text { procedures }\end{array}$ & Cost: $\$ 829.58$ & $\begin{array}{l}\text { ELIANA trial10,13 } \\
\text { CMS Physician Fee Schedule and Clinical Laboratory Fee Schedule }{ }^{18,21}\end{array}$ \\
\hline \multicolumn{3}{|l|}{ Infusion period } \\
\hline Inpatient room and board & $\begin{array}{l}\text { Proportion of patients hospitalized: } 73.42 \% \\
\text { Daily hospitalization cost: } \$ 1,323.30\end{array}$ & $\begin{array}{l}\text { ELIANA trial }{ }^{10,13} \\
\text { Kaul et al. }{ }^{19}\end{array}$ \\
\hline ICU & $\begin{array}{l}\text { Proportion of patients admitted: } 1.27 \% \\
\text { Daily ICU cost: } \$ 5,217.32\end{array}$ & $\begin{array}{l}\text { ELIANA trial }{ }^{10,13} \\
\text { Hsu et al. } 20\end{array}$ \\
\hline Physician visit & $\begin{array}{l}\text { Average number of visits: } 1 \\
\text { Unit cost: } \$ 51.90\end{array}$ & $\begin{array}{l}\text { ELIANA trial }{ }^{10,13} \\
\text { CMS Physician Fee Schedule } 18\end{array}$ \\
\hline Nurse visit & $\begin{array}{l}\text { Average number of visits: } 1 \\
\text { Unit cost: } \$ 25.95\end{array}$ & $\begin{array}{l}\text { ELIANA trial }{ }^{10,13} \\
\text { CMS Physician Fee Schedule }{ }^{18}\end{array}$ \\
\hline $\begin{array}{l}\text { Other laboratory tests and } \\
\text { procedures }\end{array}$ & Cost: $\$ 400.08$ & $\begin{array}{l}\text { ELIANA trial }{ }^{10,13} \\
\text { CMS Physician Fee Schedule and Clinical Laboratory Fee Schedule }{ }^{18,21}\end{array}$ \\
\hline \multicolumn{3}{|l|}{ Follow-up period } \\
\hline Inpatient room and board & $\begin{array}{l}\text { Month 1: } \\
\text { Proportion of patients hospitalized: } 73.42 \% \\
\text { Average LOS: } 27.3 \text { days } \\
\text { Month 2: } \\
\text { Proportion of patients hospitalized: } 8.86 \% \\
\text { Average LOS: } 5.6 \text { days } \\
\text { Daily hospitalization cost: } \$ 1,323.30\end{array}$ & $\begin{array}{l}\text { ELIANA trial }{ }^{10,13} \\
\text { Kaul et al. }{ }^{19}\end{array}$ \\
\hline ICU & $\begin{array}{l}\text { Proportion of patients admitted: } 10.13 \% \\
\text { Average LOS: } 10.9 \text { days } \\
\text { Daily ICU cost: } \$ 5,217.32\end{array}$ & $\begin{array}{l}\text { ELIANA trial } 10,13 \\
\text { Hsu et al. } 20\end{array}$ \\
\hline Physician visit & $\begin{array}{l}\text { Average number of visits: } 12 \\
\text { Unit cost: } \$ 51.90\end{array}$ & $\begin{array}{l}\text { ELIANA trial10,13 } \\
\text { CMS Physician Fee Schedule }{ }^{18}\end{array}$ \\
\hline Nurse visit & $\begin{array}{l}\text { Average number of visits: } 23 \\
\text { Unit cost: } \$ 25.95\end{array}$ & $\begin{array}{l}\text { ELIANA trial }{ }^{10,13} \\
\text { CMS Physician Fee Schedule }{ }^{18}\end{array}$ \\
\hline $\begin{array}{l}\text { Other laboratory tests and } \\
\text { procedures }\end{array}$ & $\$ 1,966.79$ & $\begin{array}{l}\text { ELIANA trial }{ }^{10,13} \\
\text { CMS Physician Fee Schedule and Clinical Laboratory Fee Schedule }{ }^{18,21}\end{array}$ \\
\hline
\end{tabular}

Under the scenario that each cost component was varied, the greatest effect was seen when AE cost was varied, and the model calculated costs ranging from $\$ 109,410$ to $\$ 165,862$ aside from the tisagenlecleucel procedure and administration. The variation in inpatient and ICU admission cost had the second greatest effect; the model calculated costs ranging from
$\$ 119,118$ to $\$ 156,180$ aside from tisagenlecleucel procedure and administration. The other cost components had more limited effect on the results. The costs aside from tisagenlecleucel procedure and administration changed by less than $1 \%$ when these cost components were varied. 


\begin{tabular}{|c|c|c|}
\hline Cost Item & Inputs & Source/Notes \\
\hline \multicolumn{3}{|l|}{ CRS-related costs } \\
\hline $\begin{array}{l}\text { Tocilizumab for } \\
\text { grade } 3 \text { or } 4 \text { CRS }\end{array}$ & $\begin{array}{l}\text { Proportion of patients: } \\
39.24 \% \\
\text { Drug cost: } \$ 3,470.49 \\
\text { Administration cost: } \\
\$ 216.93\end{array}$ & $\begin{array}{l}\text { ELIANA trial }{ }^{10,13} \\
\text { RED BOOK }{ }^{17} \\
\text { CMS Physician Fee } \\
\text { Schedule }^{18}\end{array}$ \\
\hline $\begin{array}{l}\text { ICU admission } \\
\text { for grade } 3 \text { or } 4 \\
\text { CRS }\end{array}$ & $\begin{array}{l}\text { Proportion of patients: } \\
48.10 \% \\
\text { Average LOS: } 11.2 \text { days } \\
\text { Daily ICU cost: } \$ 5,217.32\end{array}$ & $\begin{array}{l}\text { ELIANA trial10,13 } \\
\text { Hsu et al. }(2015)^{20}\end{array}$ \\
\hline \multicolumn{3}{|c|}{ B-cell aplasia-related costs } \\
\hline $\begin{array}{l}\text { IVIG for } \\
\text { B-cell aplasia }\end{array}$ & $\begin{array}{l}\text { Proportion of patients: } \\
60.76 \% \\
\text { Drug cost: } \$ 2,839.20 \\
\text { Administration cost: } \\
\$ 294.77\end{array}$ & $\begin{array}{l}\text { ELIANA trial }{ }^{10,13} \\
\text { Hettle et al. }(2015)^{37} \\
\text { RED BOOK }{ }^{17} \\
\text { CMS Physician Fee } \\
\text { Schedule }\end{array}$ \\
\hline \multicolumn{3}{|l|}{ Other AE costs } \\
\hline $\begin{array}{l}\text { AE management } \\
\text { for other grade } 3 \\
\text { or } 4 \text { AEs }\end{array}$ & Cost: $\$ 39,548.84$ & $\begin{array}{l}\text { HCUP inpatient data- } \\
\text { base }^{23,26} \\
\text { ELIANA trial } 10,13\end{array}$ \\
\hline \multicolumn{3}{|c|}{$\begin{array}{l}A E=\text { adverse event } ; C M S=\text { Centers for Medicare } E \text { Medicaid Services; } C R S=\text { cyto- } \\
\text { kine release syndrome; HCUP = Healthcare Cost and Utilization Project; } \\
\text { ICU = intensive care unit; IVIG = intravenous immunoglobulin; LOS=length of stay. }\end{array}$} \\
\hline
\end{tabular}

Under the scenario that used Grupp et al. to inform grade 3/4 CRS, IVIG use, and tocilizumab use, the model calculated a cost of $\$ 116,699$ aside from the tisagenlecleucel procedure and administration, which included $\$ 50,031$ (42.8\%) for AE management, $\$ 57,952$ (49.7\%) for inpatient and ICU admissions not attributed to AEs, \$5,209 (4.5\%) for laboratory tests and procedures, $\$ 1,780$ (1.5\%) for medical professional visits, and $\$ 1,727$ (1.5\%) for lymphodepleting drugs and their administration.

Under the scenario that used data from the HCUP database to estimate daily hospitalization and ICU costs, the model calculated a cost of $\$ 158,168$ aside from the tisagenlecleucel procedure and administration, which included $\$ 69,460$ (43.9\%) for AE management, $\$ 79,992$ (50.6\%) for inpatient and ICU admissions not attributed to AEs, \$5,209 (3.3\%) for laboratory tests and procedures, $\$ 1,780$ (1.1\%) for medical professional visits, and $\$ 1,727$ (1.1\%) for lymphodepleting drugs and their administration.

\section{Discussion}

To our knowledge, this is the first study using resource utilization data of tisagenlecleucel treatment from the ELIANA trial to provide total estimated costs and detailed breakdowns by cost component and treatment period of CAR-T immunotherapy for patients with $\mathrm{r} / \mathrm{r}$ ALL in the United States. In this economic model, we estimated a total cost of $\$ 612,779$ associated with tisagenlecleucel treatment from leukapheresis to 2 months postinfusion among pediatric and young adult patients with r/r ALL, from a U.S. hospital's perspective, of which $\$ 137,636$ $(22.5 \%)$ was in addition to the list WAC price of tisagenlecleucel $(\$ 475,000)$ and the associated administration cost $(\$ 143.08)$. The top 3 drivers of the additional cost were AE management (51.6\%), inpatient and ICU admissions not attributed to AEs (42.1\%), and laboratory tests and procedures (3.8\%).

This economic modeling study makes several important contributions to the literature. It is the first U.S.-focused study that comprehensively evaluated the health care costs associated with tisagenlecleucel treatment based on HCRU data from a clinical trial. The total estimated costs covered multiple cost components and were summarized within different periods of care from a U.S. hospital's perspective. Additionally, the model also explored various treatment scenarios to provide a plausible range of the cost estimate by varying key inputs and assumptions. Given the scarcity of comprehensive economic evaluations of novel CAR-T therapies in the literature, the cost estimates provided in the current study shed light on the economic outcomes of tisagenlecleucel for patients with $\mathrm{r} / \mathrm{r}$ ALL in the context of robust clinical outcomes observed in the clinical trial setting.

The cost estimates from this study are largely consistent with prior published economic studies of tisagenlecleucel for the treatment of pediatric and young adult patients with $\mathrm{r} / \mathrm{r}$ ALL. In 1 study that evaluated the total costs of CAR-T immunotherapy, Hernandez et al. (2018) reported a mean expected cost of $\$ 510,963$ for patients with $\mathrm{r} / \mathrm{r}$ pediatric ALL treated with tisagenlecleucel in the United States. ${ }^{24}$ This estimate is lower than that reported in the current study, which may be explained by differing cost components explored in the 2 studies. Hernandez et al. only considered a subset of the cost components evaluated in the current model (e.g., physician and facility costs for leukapheresis, lymphodepleting therapy, and CAR-T immunotherapy; drug costs; and facility costs of CRS-related hospitalizations), and did not account for key resource-use categories related to hospitalization and other important AEs, such as B-cell aplasia. Moreover, Hernandez et al. estimated resource use based on the public data source of the ELIANA trial. ${ }^{24}$

In contrast, the current study analyzed the patient-level data from the ELIANA trial to more comprehensively incorporate resource use and costs related to tisagenlecleucel treatment over the entire treatment and postinfusion period, resulting in a slightly higher total cost estimate of $\$ 612,779$. The total cost of tisagenlecleucel was also evaluated in 2 published costeffectiveness analysis studies from the U.S. payer's perspective, though these studies evaluated tisagenlecleucel costs from different time horizons and considered variable cost components and assumptions based on different study focuses. ${ }^{25,26}$ 


\section{TABLE 4 Results of Tisagenlecleucel-Associated Costs by Treatment Period and Scenario}

\begin{tabular}{|c|c|c|c|c|}
\hline & \multicolumn{3}{|c|}{ Cost by Treatment Period, $\$$} & \multirow[b]{2}{*}{ Total Cost, \$ } \\
\hline & Pretreatment & Infusion & Follow-Up & \\
\hline \multicolumn{5}{|l|}{ Base-case results } \\
\hline Total costs & 29,002 & 476,659 & 107,118 & 612,779 \\
\hline \multicolumn{5}{|l|}{ Drug and administration } \\
\hline Tisagenlecleucel procedures and administration & NA & 475,143 & NA & 475,143 \\
\hline Lymphodepleting regimens and administration & 1,727 & NA & NA & 1,727 \\
\hline Inpatient and ICU admissions & 23,966 & 1,038 & 32,949 & 57,952 \\
\hline Medical professional visits & 467 & 78 & 1,235 & 1,780 \\
\hline Laboratory tests and procedures & 2,842 & 400 & 1,967 & 5,209 \\
\hline AE management & NA & NA & 70,968 & 70,968 \\
\hline \multicolumn{5}{|l|}{ Scenario analysis results } \\
\hline Scenario $1^{a}$ outpatient setting + no AE & 4,725 & 475,621 & 2,823 & 483,169 \\
\hline Scenario $2^{\mathrm{b}}$ inpatient setting + grade $3 / 4$ CRS + B-cell aplasia & 37,934 & 476,994 & 157,446 & 672,373 \\
\hline \multicolumn{5}{|c|}{ Scenario 3 considering variation of individual cost components ( $95 \%$ CI or $\pm 25 \%$ ) } \\
\hline Lymphodepleting regimens and administration costs (-25\%) & 28,570 & 476,659 & 107,118 & 612,347 \\
\hline Lymphodepleting regimens and administration costs $(+25 \%)$ & 29,434 & 476,659 & 107,118 & 613,210 \\
\hline Inpatient and ICU admissions cost with 95\% CI lower bound & 22,212 & 476,659 & 95,390 & 594,261 \\
\hline Inpatient and ICU admissions cost with 95\% CI upper bound & 35,818 & 476,659 & 118,847 & 631,323 \\
\hline Medical professional visits cost (-25\%) & 28,885 & 476,639 & 106,810 & 612,334 \\
\hline Medical professional visits cost $(+25 \%)$ & 29,119 & 476,678 & 107,427 & 613,224 \\
\hline Laboratory tests and procedures cost $(-25 \%)$ & 28,291 & 476,559 & 106,627 & 611,476 \\
\hline Laboratory tests and procedures cost $(+25 \%)$ & 29,712 & 476,759 & 107,610 & 614,081 \\
\hline AE cost with $95 \%$ CI lower bound & 29,002 & 476,659 & 78,892 & 584,553 \\
\hline AE cost with $95 \%$ CI upper bound & 29,002 & 476,659 & 135,344 & 641,005 \\
\hline $\begin{array}{l}\text { Scenario } 4^{c} \text { using Grupp et al. to inform grade } 3 / 4 \text { CRS, IVIG use, and } \\
\text { tocilizumab use }\end{array}$ & 29,002 & 476,659 & 86,182 & 591,842 \\
\hline $\begin{array}{l}\text { Scenario } 5 \text { estimating the daily hospitalization and ICU costs based on } \\
\text { HCUP database }\end{array}$ & 37,483 & 477,133 & 118,695 & 633,311 \\
\hline \multicolumn{5}{|c|}{$\begin{array}{l}\text { aScenario } 1 \text { assumed 100\% of patients received tisagenlecleucel in the outpatient setting without experiencing any AEs during the follow-up period. } \\
\text { bScenario } 2 \text { assumed 100\% of patients received tisagenlecleucel in the inpatient setting and experienced CRS and B-cell aplasia, which required IVIG. } \\
\text { 'Scenario } 4 \text { used Grupp et al. (a real-world study published in 201914) to inform rates of AE. Detailed inputs from Grupp et al. can be found in Appendix A. } \\
\text { AE=adverse event; CI=confidence interval; CRS=cytokine release syndrome; HCUP=Healthcare Cost and Utilization Project; ICU=intensive care unit; } \\
\text { IVIG=intravenous immunoglobulin; NA=not applicable. }\end{array}$} \\
\hline
\end{tabular}

The overall cost estimates, ranging from $\$ 548,000$ to $\$ 666,754$, were within a similar range of the current study, and in both studies, the majority of the estimated cost was related to the tisagenlecleucel procedure (71\%-87\%). ${ }^{25,26}$ Both studies demonstrated the cost-effectiveness of tisagenlecleucel, with incremental cost-effectiveness ratios of $\$ 45,871$ to $\$ 61,000$ per quality-adjusted life-year gained compared with standard treatments.

For the treatment of $\mathrm{r} / \mathrm{r}$ ALL, the total cost of care associated with tisagenlecleucel was estimated to be $\$ 612,779$ in this study. This estimated cost is similar to the total reported 100-day cost associated with allogeneic stem cell transplantation (SCT; \$575,625-\$677,036, 2019 USD), which was the only potentially curative option for pediatric and young adult patients with $\mathrm{r} / \mathrm{r}$ ALL before the introduction of tisagenlecleucel. ${ }^{27,28}$ Allogeneic HSCT is currently recommended as part of a treatment option for pediatric patients with $\mathrm{r} / \mathrm{r}$ ALL receiving conventional treatment, though its role following tisagenlecleucel is unclear. ${ }^{\top}$ No head-to-head comparisons between CAR-T and HSCT have been conducted. The median survival after allogeneic HSCT for pediatric and young adult patients with $\mathrm{r} / \mathrm{r}$ ALL in first or later relapse ranges from 7.4 months to 15 months in the literature. ${ }^{29-31}$ The median OS of tisagenlecleucel was 19.1 months in the ELIANA trial. ${ }^{10}$ However, it is important to note that these survival estimates for HSCT were derived from single-arm studies with populations that are not fully comparable to the ELIANA population; in general, there is limited evidence on HSCT efficacy in pediatric and young adult patients in second or later relapse.

Allogeneic HSCT is also not suitable for all patients; poor health or presence of comorbidities may preclude its use, and a suitably matched sibling donor is available in only $30 \%$ of cases. $^{32,33}$ In cases where allogeneic HSCT is possible, however, the treatment has been associated with high rates of 
complications, some of which require years to resolve. Indeed, in the study conducted by Crotta et al. (2018), 57\% and 35\% of patients experienced acute and chronic graft versus host disease, respectively, after the allogeneic HSCT procedure. Additionally, a small fraction of patients developed severe secondary malignancies such as acute myeloid leukemia and lymphoproliferative disease. ${ }^{29}$ While tisagenlecleucel treatment is associated with unique AEs, most AEs observed in the ELIANA trial were resolved within a few months after infusion. ${ }^{10}$ However, additional long-term, real-world data are necessary to continue monitoring the situation and to improve our understanding of the safety profile of CAR-T therapy. Additionally, comparative studies on clinical outcomes and costs of tisagenlecleucel and allogeneic HSCT are needed to compare these 2 treatments.

An important component of the overall cost of treatment is the incremental cost of care in addition to the list WAC price and administration cost of tisagenlecleucel. This component was estimated to be $\$ 137,636$ in the current study, ranging from $\$ 8,026$ to $\$ 197,230$ depending on the hospitalization and AE assumptions. In absolute terms, these additional estimated costs are lower compared with the expected medical expenses incurred by pediatric and young adult patients with $\mathrm{r} / \mathrm{r}$ ALL receiving alternative treatments. Existing modeling studies have estimated the costs of other currently available chemotherapy or targeted treatments, with estimates ranging from $\$ 238,000$ to $\$ 307,000$ in addition to the drug costs. ${ }^{26}$

With the continued advancement of innovative cell and gene therapies that offer improved clinical outcomes, the composition of overall cost of patient care may shift with drug/procedure cost becoming the major component of the overall treatment cost. Indeed, the WAC price of tisagenlecleucel and its associated administration cost comprised $77.5 \%$ of the total health care cost of tisagenlecleucel treatment in this study. Similarly, based on a cost-effectiveness analysis, the list price of the new gene therapy onasemnogene abeparvovec contributed $57 \%$ to $73 \%$ of the total cost of care for pediatric patients with spinal muscular atrophy type 1 , depending on various pricing assumptions. ${ }^{34}$ Finally, the gene therapy voretigene neparvovec accounted for $82 \%$ of the total health care costs for patients with inherited retinal disorder in a cost-utility study. ${ }^{35}$

\section{Limitations}

The results of this study should be interpreted in light of some limitations. First, HCRU among patients receiving tisagenlecleucel treatment was estimated based on clinical trial data, which may not represent the real-world HCRU with a more heterogeneous patient population (e.g., patients with multiple comorbidities) and practice patterns (e.g., management of CRS and hospitalization duration). A scenario analysis was conducted using CRS rate as well as tocilizumab and IVIG utilization information reported in a real-world study by Grupp et al. with a median follow-up of 6 months. ${ }^{14}$ Overall, the study reported lower rates of grade 3 and above CRS (14\% vs. 48\% from ELIANA) as well as utilization of tocilizumab and IVIG treatment compared with the ELIANA trial. ${ }^{14}$ This was likely driven by the different grading system and management of CRS in real-world practice from those used in clinical trials as physicians gained more experience with CAR-T treatment. The ELIANA trial used the Penn grading system to classify CRS grade, which tends to assign a higher grade of CRS compared with the ASTCT criteria more commonly adopted in real-world practice. ${ }^{14,22}$ However, there is currently a lack of real-world evidence to inform HCRU and costs associated with other cost components (e.g., hospitalization, medication use, etc.) for pediatric patients with tisagenlecleucel infusion. ${ }^{22}$ With a realworld study currently underway, future analyses may provide updated insight into the HCRU and costs of tisagenlecleucel treatment in real-world clinical practice. ${ }^{14}$

Second, our analysis was limited to 2 months of follow-up post-tisagenlecleucel infusion, which does not capture longterm safety outcomes associated with the treatment. Therefore, our estimated cost of care in addition to the tisagenlecleucel procedure may be underestimated. However, the total estimated cost of care from the current model was similar to the total costs estimated in the prior cost-effectiveness studies with longer time horizons (e.g., lifetime), which suggests that our current estimates may still capture the majority of the costs associated with tisagenlecleucel treatment. ${ }^{25,26}$

Finally, our estimated costs may not reflect the true costs incurred by hospitals because of the lack of real-world data and use of publicly available databases. For example, we used the WAC in RED BOOK to estimate drug costs, which may not represent the true costs incurred by hospitals or how much hospitals get reimbursed. Indeed, under CMS, hospitals will only receive $\$ 242,450$ payment in 2020 for CAR-T therapy, which is much lower than the list price of CAR-T therapy, but the payment amount does not necessarily reflect how much the hospital has paid for tisagenlecleucel treatment either. ${ }^{36}$ Future research with long-term, real-world, follow-up data will be important to provide more accurate and refined cost estimates of tisagenlecleucel treatment in clinical practice.

\section{Conclusions}

In this economic model, tisagenlecleucel treatment among pediatric and young adult patients with $\mathrm{r} / \mathrm{r}$ ALL was estimated to cost $\$ 612,779$. The cost of care in addition to the price of tisagenlecleucel accounted for $22.5 \%$ of the total, with AE management and inpatient and ICU admissions being main drivers. While these results provide valuable economic information regarding tisagenlecleucel therapy among pediatric and young adult patients with $\mathrm{r} / \mathrm{r}$ ALL from a U.S. hospital's perspective, additional analyses are warranted to assess the cost of CAR-T therapy in the context of current standards of care in realworld clinical practice. 


\section{Authors}

HONGBO YANG, PhD; CYNTHIA Z. शI, MBA; XINGLEI CHAI, PhD; and ERIC Q. WU, PhD, Analysis Group, Boston, Massachusetts. YANNI HAO, PhD, Novartis Pharmaceuticals Corporation, East Hanover, New Jersey.

AUTHOR CORRESPONDENCE: Hongbo Yang, PhD, Analysis Group, 111 Huntington Ave., 14th Fl., Boston, MA 02199.

Tel.: 617.425.8175; Email: Hongbo.Yang@analysisgroup.com.

\section{DISCLOSURES}

This study was supported by Novartis. The study sponsor was involved in several aspects of the research, including the study design, the interpretation of data, the writing of the manuscript, and the decision to submit the manuscript for publication. Hao is an employee of Novartis and has stock/ stock options. Yang, Chai, Qi, and Wu are employees of Analysis Group, which received consulting fees from Novartis for work on this study.

Part of the material in this manuscript was presented at the American Society of Hematology Annual Meeting held December 7-10, 2019, in Orlando, FL.

\section{ACKNOWLEDGMENTS}

Medical writing support was provided by Muhan Yuan, Christine Tam, and Louise Yu, employees of Analysis Group, which received consulting fees from Novartis for work on this study.

\section{REFERENCES}

1. Surveillance, Epidemiology, and End Results (SEER) Program. Cancer stat facts: leukemia - acute lymphocytic leukemia (ALL). Available at: https:// seer.cancer.gov/statfacts/html/alyl.html. Accessed May 27, 2020.

2. Ward E, DeSantis C, Robbins A, Kohler B, Jemal A. Childhood and adolescent cancer statistics, 2014. CA Cancer J Clin. 2014;64(2):83-103.

3. Terwilliger T, Abdul-Hay M. Acute lymphoblastic leukemia: a comprehensive review and 2017 update. Blood Cancer J. 2017;7(6):e577.

4. Gore L, Locatelli F, Zugmaier G, et al. Survival after blinatumomab treatment in pediatric patients with relapsed/refractory B-cell precursor acute lymphoblastic leukemia. Blood Cancer J. 2018;8(9):80

5. Hijiya N, Thomson B, Isakoff MS, et al. Phase 2 trial of clofarabine in combination with etoposide and cyclophosphamide in pediatric patients with refractory or relapsed acute lymphoblastic leukemia. Blood. 2011;118(23):6043-49

6. Jeha S, Gaynon PS, Razzouk BI, et al. Phase II study of clofarabine in pediatric patients with refractory or relapsed acute lymphoblastic leukemia. J Clin Oncol. 2006;24(12):1917-23.

7. National Comprehensive Cancer Network. NCCN Clinical Practice Guidelines in Oncology (NCCN Guidelines) pediatric acute lymphoblastic leukemia. Version 1.2020. 2019. Available at: https://www.nccn.org/ professionals/physician_gls/pdf/ped_all.pdf. Accessed May 27, 2020.

8. Nguyen K, Devidas M, Cheng SC, et al. Factors influencing survival after relapse from acute lymphoblastic leukemia: a Children's Oncology Group study. Leukemia. 2008;22(12):2142-50.

9. von Stackelberg A, Locatelli F, Zugmaier G, et al. Phase I/phase II study of blinatumomab in pediatric patients with relapsed/refractory acute lymphoblastic leukemia. J Clin Oncol. 2016;34(36):4381-89.
10. Maude SL, Laetsch TW, Buechner J, et al. Tisagenlecleucel in children and young adults with B-cell lymphoblastic leukemia. N Engl J Med. 2018;378(5):439-48.

11. Kymriah (tisagenlecleucel) suspension for intravenous infusion. Novartis Pharmaceuticals Corporation. May 2018. Available at: https://www.fda.gov/ files/vaccines\%2C\%20blood\%20\%26\%20biologics/published/Package-Insert--KYMRIAH.pdf. Accessed May 27, 2020.

12. Yakoub-Agha I, Chabannon C, Bader P, et al. Management of adults and children undergoing chimeric antigen receptor T-cell therapy: best practice recommendations of the European Society for Blood and Marrow Transplantation (EBMT) and the Joint Accreditation Committee of ISCT and EBMT (JACIE). Haematologica. 2020;105(2):297-316.

13. Determine efficacy and safety of CTLO19 in pediatric patients with relapsed and refractory B-cell ALL and high risk B-cell ALL at first relapse. Determine feasibility and safety of CTLO19 therapy in pediatric patients with high risk B-cell ALL that relapsed $<6$ months post ALL-HSCT (ELIANA). ClinicalTrials.gov identifier: NCT02435849. Updated April 28, 2020. Available at: https://clinicaltrials.gov/ct2/show/NCT02435849. Accessed May 27, 2020.

14. Grupp S, Hu Z-H, Zhang Y, et al. Tisagenlecleucel chimeric antigen receptor (CAR) T-cell therapy for relapsed/refractory children and young adults with acute lymphoblastic leukemia (ALL): real world experience from the Center for International Blood and Marrow Transplant Research (CIBMTR) and cellular therapy (CT) registry. Blood. 2019;134(suppl 1):2619.

15. Agency for Healthcare Research and Quality. HCUP fast stats - trends in inpatient stays. May 2020. Available at: https://www.hcup-us.ahrq.gov/faststats/NationalTrendsServlet? measure $1=02 \&$ characteristicl $=02 \&$ time $1=10 \&$ measure $2=\&$ characteristic $2=01 \&$ time $2=10$ \&expansionInfoState $=$ hide $\&$ dataT ablesState $=$ hide $\&$ definitions State $=$ hide\&exportState $=$ hide. Accessed May 27, 2020.

16. Barrett ML, Smith MW, Elixhauser A, Honigman LS, Pines JM. Utilization of intensive care services, 2011. HCUP Statistical Brief \#185. December 2014. Available at: https://www.hcup-us.ahrq.gov/reports/ statbriefs/sb185-Hospital-Intensive-Care-Units-2011.pdf. Accessed May 27, 2020.

17. IBM Watson Health. Micromedex RED BOOK Online. Database. 2019. Available at: https://www.ibm.com/products/micromedex-with-watson. Accessed May 27, 2020.

18. Centers for Medicare \& Medicaid Services. Physician fee schedule. Updated April 3, 2020. Available at: http://www.cms.gov/apps/physician-feeschedule/overview.aspx. Accessed May 30, 2019.

19. Kaul S, Korgenski EK, Ying J, et al. A retrospective analysis of treatmentrelated hospitalization costs of pediatric, adolescent, and young adult acute lymphoblastic leukemia. Cancer Med. 2016;5(2):221-29.

20. Hsu BS, Brazelton TB III. A comparison of costs between medical and surgical patients in an academic pediatric intensive care unit. WMJ. 2015;114(6):236-39

21. Centers for Medicare \& Medicaid Services. Clinical laboratory fee schedule. Updated January 2, 2020. Available at: https://www.cms.gov/ Medicare/Medicare-Fee-for-Service-Payment/ClinicalLabFeeSched/index. html. Accessed May 27, 2020.

22. Lee DW, Santomasso BD, Locke FL, et al. ASTCT consensus grading for cytokine release syndrome and neurologic toxicity associated with immune effector cells. Biol Blood Marrow Transplant. 2019;25(4):625-38.

23. Agency for Healthcare Research and Quality. HCUP Kids' Inpatient Database. September 2019. Available at: https://www.hcup-us.ahrq.gov/db/ nation/kid/kiddbdocumentation.jsp. Accessed May 27, 2020.

24. Hernandez I, Prasad V, Gellad WF. Total costs of chimeric antigen receptor T-cell immunotherapy. JAMA Oncol. 2018;4(7):994-96. 
25. Institute for Clinical and Economic Review. Chimeric antigen receptor T-cell therapy for B-cell cancers: effectiveness and value. March 23, 2018. Available at: https://icer-review.org/wp-content/uploads/2017/07/ICER_ CAR_T_Final_Evidence_Report_032318.pdf. Accessed May 27, 2020.

26. Lin JK, Lerman BJ, Barnes JI, et al. Cost effectiveness of chimeric antigen receptor T-cell therapy in relapsed or refractory pediatric B-cell acute lymphoblastic leukemia. J Clin Oncol. 2018;36(32):3192-202.

27. Broder MS, Quock TP, Chang E, et al. The cost of hematopoietic stem-cell transplantation in the United States. Am Health Drug Benefits. 2017;10(7):366-74

28. Maziarz RT, Guerin A, Gauthier G, et al. Five-year direct costs of acute lymphoblastic leukemia pediatric patients undergoing allogeneic stem cell transplant. Int J Hematol Oncol. 2016;5(2):63-75.

29. Crotta A, Zhang J, Keir C. Survival after stem-cell transplant in pediatric and young-adult patients with relapsed and refractory B-cell acute lymphoblastic leukemia. Curr Med Res Opin. 2018;34(3):435-40.

30. Kato M, Horikoshi Y, Okamoto Y, et al. Second allogeneic hematopoietic SCT for relapsed ALL in children. Bone Marrow Transplant. 2012;47(10):1307-11.

31. Yaniv I, Krauss AC, Beohou E, et al. Second hematopoietic stem cell transplantation for post-transplantation relapsed acute leukemia in children: a retrospective EBMT-PDWP study. Biol Blood Marrow Transplant. 2018;24(8):1629-42.

32. Ballen KK, Gluckman E, Broxmeyer HE. Umbilical cord blood transplantation: the first 25 years and beyond. Blood. 2013;122(4):491-98.
33. Nietfeld JJ, Pasquini MC, Logan BR, Verter F, Horowitz MM. Lifetime probabilities of hematopoietic stem cell transplantation in the U.S. Biol Blood Marrow Transplant. 2008;14(3):316-22.

34. Malone DC, Dean R, Arjunji R, et al. Cost-effectiveness analysis of using onasemnogene abeparvocec (AVXS-101) in spinal muscular atrophy type 1 patients. J Mark Access Health Policy. 2019;7(1):1601484

35. Zimmermann M, Lubinga SJ, Banken R, et al. Cost utility of voretigene neparvovec for biallelic RPE65-mediated inherited retinal disease. Value Health. 2019;22(2):161-67.

36. Centers for Medicare \& Medicaid Services. Fiscal year (FY) 2020 Medicare Hospital Inpatient Prospective Payment System (IPPS) and Long Term Acute Care Hospital (LTCH) Prospective Payment System proposed rule and request for information. Fact sheet. April 23, 2019. Available at: https://www.cms.gov/newsroom/fact-sheets/fiscal-year-fy-2020-medicarehospital-inpatient-prospective-payment-system-ipps-and-long-term-acute. Accessed May 27, 2020

37. Hettle R, Corbett M, Hinde S, et al. The assessment and appraisal of regenerative medicines and cell therapy products: an exploration of methods for review, economic evaluation and appraisal. Health Technol Assess. 2015;21(7):1-204.

38. Mueller EL, Walkovich KJ, Mody R, Gebremariam A, Davis MM. Hospital discharges for fever and neutropenia in pediatric cancer patients: United States, 2009. BMC Cancer. 2015;15:388. 
Estimation of Total Costs in Pediatric and Young Adult Patients with Relapsed or

Refractory Acute Lymphoblastic Leukemia Receiving Tisagenlecleucel from a U.S. Hospital's Perspective

\begin{tabular}{|c|c|}
\hline \multicolumn{2}{|c|}{$\begin{array}{ll}\text { APPENDIX A } & \text { CRS and IVIG and Tocilizumab Use for } \\
& \text { Tisagenlecleucel Based on Grupp et al. }\end{array}$} \\
\hline rameter & $\%$ \\
\hline ts with grade 3 ar & 4 \\
\hline roportion of patients treated with tociliz & 3 \\
\hline roportion of patients treated with IVIG & 41 \\
\hline \multicolumn{2}{|c|}{$\begin{array}{l}\text { Note: Grupp et al. (2019)14 analyzed real-world data from patients with relapsed/ } \\
\text { refractory B-cell pediatric ALL who received tisagenlecleucel from } 45 \text { centers in the } \\
\text { United States through the Center for International Blood and Marrow Transplant } \\
\text { Research Cellular Therapy registry (data cutoff September 2019). Safety outcomes } \\
\text { were available from } 154 \text { patients, who had at least } 3 \text { months of follow-up after } \\
\text { infusion. Median follow-up time was } 6 \text { months (range: <1-14 months) among } \\
\text { survivors. The American Society for Transplantation and Cellular Therapy } \\
\text { consensus criteria were used to assess and grade CRS. } \\
\text { ALL=acute lymphoblastic leukemia; CRS = cytokine release syndrome; } \\
\text { IVIG=intravenous immunoglobulin. }\end{array}$} \\
\hline
\end{tabular}


Estimation of Total Costs in Pediatric and Young Adult Patients with Relapsed or Refractory Acute Lymphoblastic Leukemia Receiving Tisagenlecleucel from a U.S. Hospital's Perspective

\begin{tabular}{|c|c|c|c|c|}
\hline Grade 3 and 4 AEs $\geq 5 \%$ & Patients with AE, \% & Cost per Event, \$ & ICD-9-CM Code & $\begin{array}{l}\text { Sources for Unit } \\
\text { Cost }^{23,38}\end{array}$ \\
\hline Acute renal failure & 10.1 & $25,883.03$ & 790.4 & HCUP KID \\
\hline Alanine aminotransferase increased & 8.9 & $8,099.64$ & $584 . x$ & HCUP KID \\
\hline Anemia & 11.4 & $19,165.83$ & 280.x, 281.x, 284.x, 285.x & HCUP KID \\
\hline Aspartate aminotransferase increased & 13.9 & $8,099.64$ & 790.4 & HCUP KID \\
\hline Blood bilirubin increased & 11.4 & $14,324.30$ & 277.4 & HCUP KID \\
\hline Decreased appetite & 15.2 & $9,507.66$ & 783.0 & HCUP KID \\
\hline Encephalopathy & 5.1 & $20,420.96$ & 349.82 & HCUP KID \\
\hline Febrile neutropenia & 34.2 & $18,978.71$ & NA & Mueller et al. (2015) \\
\hline Hyperglycemia & 5.1 & $4,905.53$ & 790.29 & HCUP KID \\
\hline Hypertension & 5.1 & $14,110.25$ & $401 . x$ & HCUP KID \\
\hline Hypocalcemia & 6.3 & $10,344.41$ & 275.41 & HCUP KID \\
\hline Hypogammaglobulinemia & 5.1 & $12,260.13$ & 279.00 & HCUP KID \\
\hline Hypokalemia & 13.9 & $8,226.46$ & 276.8 & HCUP KID \\
\hline Hypophosphatemia & 11.4 & $15,100.60$ & 275.3 & HCUP KID \\
\hline Hypotension & 20.3 & $10,329.77$ & $458 . x$ & HCUP KID \\
\hline Hypoxia & 20.3 & $8,388.83$ & 799.02 & HCUP KID \\
\hline Lymphocyte count decreased & 19.0 & $6,013.69$ & $288.5 x$ & HCUP KID \\
\hline Neutropenia & 11.4 & $14,969.05$ & $288.0 x$ & HCUP KID \\
\hline Neutrophil count decreased & 26.6 & $14,969.05$ & $288.0 x$ & HCUP KID \\
\hline Platelet count decreased & 19.0 & $10,396.55$ & $287.3,287.4,287.5$ & HCUP KID \\
\hline Pulmonary edema & 8.9 & $12,193.56$ & $518.4,514$ & HCUP KID \\
\hline Pyrexia & 12.7 & $4,912.64$ & $780.6 x$ & HCUP KID \\
\hline Tachypnea & 5.1 & $8,045.21$ & 786.06 & HCUP KID \\
\hline Thrombocytopenia & 11.4 & $10,396.55$ & $287.3,287.4,287.5$ & HCUP KID \\
\hline White blood cell count decreased & 21.5 & $6,013.69$ & $288.5 \mathrm{x}$ & HCUP KID \\
\hline
\end{tabular}

